\title{
Imaginaire mondial d'après La théorie des nuages de Stéphane Audeguy
}

\author{
Préparé par \\ Attia Elemam Alkolaly \\ Professeur-adjoint de littérature et de \\ civilisation françaises \\ Faculté des lettres- Université de \\ Damiette
}


Imaginaire mondial d'après La théorie des nuages de Stéphane Audeguy

Dr. Attia Elemam Alkolaly

Imaginaire mondial d'après La théorie des nuages de Stéphane Audeguy

La théorie des nuages, paru en 2005 chez Gallimard, est le premier roman de Stéphane Audeguy. Ce roman a connu un succès mérité et des critiques élogieuses à sa sortie en cristallisant l'attention sur le jeune romancier. II s'agit d'une espèce d'histoire de la perception des nuages, de la généalogie des inventeurs des nuages, qui unissent leur désir à ces masses instables, source d'eau et de rêve. Précis comme un bulletin météorologique et libre comme l'air, ce roman est ainsi un résumé de poésie atmosphérique.

Le roman raconte l'histoire d'une jeune bibliothécaire, Virginie Latour, engagée par un couturier japonais installé à Paris, Akira Kumo, pour classer sa bibliothèque. Celle-ci contient surtout des ouvrages concernant la météorologie, et plus exactement les nuages. Akira Kumo entreprend de lui raconter l'histoire des nuages, et aussi celle des scientifiques qui se sont intéressés aux nuages. La nature y est très présente, mais il ne donne aucune description de nature pure. Que ce soient les nuages, un volcan du Pacifique qui se réveille, ou encore la bombe atomique d'Hiroshima qui bouleverse le destin du protagoniste principal du roman, Akira Kumo.

Stéphane Audeguy, né en 1964, enseigne l'histoire du cinéma et des arts dans un établissement public des Hauts-de-Seine. En 2007, il a reçu le prix des "Deux Magots" pour son deuxième roman intitulé Fils unique, paru en $2006 \mathrm{chez}$ Gallimard. C'est la littérature mondiale qui fascine Stéphane Audeguy, qui a beaucoup lu la littérature anglo-saxonne et la littérature japonaise surtout Murakami Kijõ, poète et écrivain japonais, né en 1865 et mort en 1938. 
La parution de La théorie du paysage en France (1995) correspond à la publication du Court traité du paysage (1997) du philosophe Alain Roger. Ces deux titres sont symptomatiques d'un intérêt pour le lieu et l'espace. Les liens entre la géographie et la littérature ont conduit à la naissance de courants nouveaux: la géopoétique, la géocritique et la géographie littéraire. Un même phénomène humain donne lieu à des exploitations différentes selon les savoirs et les cultures. Il faut se référer à la notion d'interculturalité qui s'est très vite répandue au cours de ces dernières années.

"Il y a interculturalité littéraire quand il y a transfert littéraire entre des civilisations dont les différences se révèlent d'une part dans l'utilisation de codes de communication différents et sont d'autre part le résultat d'une distance temporelle ou spatiale". (Gottingen, p:438) Chez Stéphane Audeguy, à côté de la culture, les livres rangés sur les rayons de la bibliothèque d'Akira Kumo, il y a aussi la nature, le ciel, les nuages, tout ce qui excite l'aventure. Entre ces deux données contradictoires, l'écrivain recourt à l'imagination d'une façon frappante pour réconcilier les objets. A celle-ci, il faut un objet tangible et visible, même s'il est composé d'air et d'eau, et que le ciel seul en contient l'essentiel et la forme. Notons que le mot "théorie" du titre de roman est bien choisi puisqu'il prend racine dans le fait d'observer et de concevoir. Ce titre va nous emmener plus loin, nous faire rêver, nous élever parmi des réflexions aériennes.

Grâce à ses voyages multiples en Amérique et en Afrique de l'Est, Stéphane Audeguy a pu comprendre ces régions vastes et solitaires, ces paysages sauvages, intacts et vierges de toute empreinte humaine, où les cieux sont dégagés. Ce qui l'intéresse, c'est de mettre en relief des intensités liées à la perception de la nature. Effectivement, la fusion entre l'homme et la nature est cruciale, et l'homme s'efforce de la maîtriser.

Stéphane Audeguy a justifié le choix des nuages comme "sujet" dans un entretien avec Thierry Guichard dans "Le 
Imaginaire mondial d'après La théorie des nuages de Stéphane Audeguy

Dr. Attia Elemam Alkolaly

Matricule des anges", No 101, Mars 2009 déclarant: "Pourquoi ai-je choisi ce sujet des nuages? Pour faire un roman. Bien entendu, volens nolens, on est amené à se situer. Le nuage est un non-objet. La météorologie m'intéresse parce que c'est mondial. Parler des nuages, c'est parler d'un monde fini, au sens de la finitude. Mais c'était aussi faire un roman écologique au sens très large et le mot "fini" est à entendre d'une autre façon." (Guichard, p:23)

Le choix d'un "non-objet" correspond à une sorte de flou; il établit un lien entre l'objet de l'étude et l'étude elle-même. Donc, Virginie Latour évoque le "travail nébuleux dont on lui a parlé." (La théorie des nuages, $\mathrm{p}: 33$ )

Il est vrai que le sujet du roman est original et touchant. La météorologie et ses précurseurs est un thème nouveau dans la littérature, et l'existence des passionnés des nuages est émouvante. La théorie des nuages accorde une place primordiale à la photographie; il s'agit d'établir avec la réalité un rapport non linguistique. Le texte apparaît comme une image verticale et non plus horizontale. C'est une façon de matérialiser le fait que la page n'est pas le paysage, non plus que le paysage une page.

La théorie des nuages se compose de trois parties presque équilibrées. La première partie, intitulée " $L$ 'étude des ciels", et la deuxième, intitulée "Vers d'autres latitudes", sont identiques: chacune est composée de treize séquences. Mais la troisième, intitulée "Le Protocole Abercrombie", est composée de quinze séquences. Chaque séquence est commencée par un espace blanc et terminée par un espace blanc pour donner au roman une forme poétique. Stéphane Audeguy ne définit pas la poésie par la rime, mais c'est un lien au monde qui implique composition. Dans le roman, il a essayé autant que possible de donner un système de rimes globales à l'intérieur du récit. Et tout le récit est représenté 
avec une consécution narrative, reliant la poésie à la composition du roman.

De plus, la structure du roman est influencée aussi par le mouvement instable des nuages, et Stéphane Audeguy l'a rappelé dans un entretien: "Pour La théorie des nuages, j'aurais bien aimé trouver l'équivalent de la structure d'un nuage comme structure narrative. Les ruines de ce projet dément sont liées à un désenchâssement de ce récit, composé de petites structures qui me font penser à une espèce de défilé de nuages. Les bords de ces nuages m'intéressaient aussi, comme c'est dit dans le roman. D'autre part, c'était lié pour moi aux Mille et Une Nuits, sans l'idée de raconter toutes les histoires possibles. La forme est venue de ça et du fait que j'ai combiné entre elles plusieurs histoires résumées sur des post-it." (La Femelle du requin, p:59)

Outre que cela contient de nombreuses lacunes concernant les dates, cela donne au roman une narration fragmentée. L'histoire de Luke Howard, commencée dans la première séquence de la première partie du roman, est coupée par l'histoire de Virginie Latour et Akira Kumo et aussi par celle du peintre Carmichael. On trouve, dans la première partie du roman, le début des révélations concernant le passé d'Akira Kumo à Hiroshima, qui se complètent dans la troisième partie. En revanche, le récit du Congrès météorologique de 1889 , qui se déroule sur plusieurs séquences, n'est pas morcelé.

Cette structure du roman a une fonction dans l'histoire ellemême: Akira Kumo voit que le travail de Virginie Latour développe vite, et lui raconte des histoires qu'il laisse en suspens. Même quand elle était à Londres et qu'il lui écrivait ses souvenirs sur Hiroshima, il le faisait en deux lettres distinctes malgré la distance. Le long récit qui est fait sans cesse, celui du Congrès de 1889, est raconté par le petit-fils d'Abercrombie à Virginie Latour. Il n'y a pas entre eux la relation d'amitié concernant les nuages, et donc ni d'échange narratif. L'échange narratif entre Virginie Latour et Akira Kumo évolue April 2016 
Imaginaire mondial d'après La théorie des nuages de Stéphane Audeguy

Dr. Attia Elemam Alkolaly

au fil de l'histoire. C'est Virginie Latour qui peu à peu commence à raconter, et Akira Kumo attend ses paroles impatiemment:

“Quand Virginie Latour n'est pas à ses côtés, quand Virginie Latour n'est pas là pour lui parler des atolls du Pacifique et de Richard Abercrombie, Akira Kumo s'ennuie ferme." (La théorie des nuages, $\mathrm{p}$ :258) Et après le trépas d'Akira Kumo, Virginie Latour s'occupe à la publication du Protocole Abercrombie en pensant à son ami:

"Quand elle écrit, pour se donner du courage elle s'imagine qu'elle parle à Akira, dans la bibliothèque de la rue Lamarck." (p:276) Bref, la structure du roman se distingue par l'évolution des rapports entre les protagonistes, et donne de l'histoire une image à la fois dispersée et d'une grande unité: une forme de déchirure nuageuse comme si le récit ressemble à des nuages qui s'assemblent. Virginie Latour écoute les histoires relatées par Akira Kumo, et Stéphane Audeguy, à son tour, raconte au lecteur ces mêmes histoires. La narration de différents épisodes des passionnés de nuages se déroule sans guillemets ni espace blanc, avec un caractère de discours rapporté, rappelé par quelques incises et par des répétitives: le verbe "dire" revient à plusieurs reprises, et l'expression "dit Akira Kumo à Virginie Latour" deux fois. (p:49-98)

De même, la rencontre de deux personnages principaux, Akira Kumo et Virginie Latour, forme le fil narratif essentiel du roman, ainsi que ses effets sur leurs destins respectifs. Ce discours rapporté fait de Virginie Latour une narrataire et en même temps, elle devient à son tour une narratrice auprès d'Akira Kumo à la fin du roman en racontant les aventures d'Abercrombie. Au début du roman, Akira Kumo raconte à Virginie Latour des histoires qu' "on ne peut pas dire qu'elles soient fausses, on ne peut pas dire qu'il les fabrique de toutes 
pièces; mais il est évident qu'il les aménage, qu'il y ajoute des éléments [...] Virginie Latour a réfléchi à tout ça; elle a conclu que ça n'avait pas d'importance" (p:79-80), et le lecteur est invité aussi à écouter ces histoires "avec un plaisir sans mélange". (p:80)

Ici, on pense au Bartlebooth de La vie mode d'emploi, et divers aspects rapprochent ce roman de Georges Perec: le goût du faux et des personnages entêtés, une anglophilie exagérée que l'on remarque quand Stéphane Audeguy parle en traducteur littéral de "rivière Tamise". L'auteur mélange également personnages et faits vrais ou faux avec une écriture magnétique.

Pour le romancier comme pour son personnage, peu importe parfois que ce que l'on raconte soit vrai ou faux. Chez Stéphane Audeguy, le mélange du réel et de l'imaginaire est aussi consacré au personnage auquel la narration est essentiellement attribuée: "Akira Kumo [...] ne déteste pas mentir, du moment que le mensonge porte une vérité supérieure à celle des faits objectivement constatables." (La théorie des nuages, $\mathrm{p}: 42$ )

Stéphane Audeguy s'occupe beaucoup de raconter et d'écouter des histoires. Et cet intérêt suscite l'invention, les "aménagements". Akira Kumo raconte longuement la rencontre entre Luke Howard, l'inventeur de la classification des nuages et Goethe, romancier allemand passionné par les sciences, rencontre dont aucun témoignage n'est donné comme certaine.

De plus, Akira Kumo relate les aventures de personnes historiques en mêlant le vrai et le faux. Dans "Interview de Stéphane Audeguy", l'écrivain a avoué à Shadi Biglarzadeh qu'il a modifié certaines biographies, comme celle d'Abercrombie, qui n'a jamais écrit le Protocole que le roman lui attribue, et en a créé d'autres, ainsi celle du peintre Carmichael, inspiré de peintre anglais John Constable [1776-1837], alors qu'il n'a pas changé la vie de Luke Howard. Stéphane Audeguy, combinant vrai et faux, ne cherche pas à aberrer son lecteur, ni à le faire douter. Il a déclaré qu'il recherchait plutôt l'exactitude que la 
Imaginaire mondial d'après La théorie des nuages de Stéphane Audeguy

Dr. Attia Elemam Alkolaly

vérité: "J'espère que tout est le plus exact possible, ce qui est pour moi plus important que l'opposition vrai-faux..." (alalettre.com/interview-Stephane.Audeguy.php (consulté le 15 juin 2011)

De plus, il donne, dans son roman, cet intérêt à Akira Kumo: l'exactitude semble correspondre à cette "vérité supérieure à celle des faits objectivement constatables" que rechercherait le personnage. L'objectif que Stéphane Audeguy chercherait dans le roman consiste à présenter l'essentiel de la réalité. Cela convient à la manière schématique qui distingue les personnages. Ce schéma correspond à un refus de l'analyse psychologique des personnages. Il dit de Richard Abercrombie:

"Il n'aime pas la psychologie. Il pense qu'il ne faut pas expliquer les gens, jamais, qu'on ne peut pas faire le tour d'un homme comme on fait celui d'un monument, qu'il faut laisser les gens tranquilles, même quand ils sont morts." (La théorie des nuages, $p: 247$ )

La plupart des personnages du roman, inventés ou réels, sont des observateurs, des hommes méditatifs et discrets, un peu distraits, qui poursuivent leurs chimères. Ils vivent en marge du monde, dans un temps de l'invention scientifique que le temps du baptême. A titre d'exemple, Luke Howard, un talent anglais, dévot, pharmacien, lecteur passionné de la Bible et classificateur des nuages au début du XIXe siècle "car les noms de baptême des choses ne fonctionnent pas comme ceux des hommes [...] Elles existent en dehors de leur nom; elles peuvent exister pendant des siècles, muettes et innommées", (p:16-17) ou Richard Abercrombie, amateur de la science météorologique qui se trouve en septembre 1890 dans la forêt de Bornéo, attaqué par des sangsues, et qui continue sa méditation du ciel. Stéphane Audeguy présente une autre généalogie, un autre temps très contemporain, puisqu'il nous emmène jusqu'à l'été 2006. Deux 
personnages le fréquentent: Akira Kumo et son traumatisme, et Virginie Latour et sa découverte d'elle-même.

En fait, le roman présente une sélection de personnages divers. Et l'intertextualité est un aspect dominant de ce texte. Nous trouvons une correspondance entre Sur la modification des nuages de Luke Howard, Sky Studies de Carmichael, et Recherches météorologiques de Cecil Scott Forester, écrivain britanique [1899-1966]. L'intrigue dépend de la recherche, exécutée par Akira Kumo, d'un texte qui l'attire depuis longtemps: l'unique exemplaire du "Protocole Abercrombie".

Les lieux sont aussi variés dans le roman. On passe du balcon d'Akira Kumo dans une rue de Paris, au paysage de Hampstead, à Hiroshima où la bombe atomique vient d'exploser. L'écrivain énumère les effets météorologiques de l'explosion du volcan de Krakatoa en 1883 en Indonésie, et les répercussions graves de ce phénomène sur l'être humain. Carmichael observe que "Le ciel n'est pas un objet, c'est un milieu, et un milieu sauvage". Et l'écrivain présente ce milieu sauvage du ciel d'une façon bien orchestrée.

La théorie des nuages essaye à produire des mots clés, à présenter l'histoire, tout en interrogeant le mystère des passions humaines. Il mélange le savoir à la fiction, à tel point que le lecteur ne doit plus interroger la vérité des références à des personnages historiques. Le roman s'attache à la fiction qui le constitue, en des passages métatextuels qui conduisent le lecteur à s'interroger sur ses pouvoirs. Ce retour de la fiction distingue le roman contemporain, et le roman élargit l'horizon de la fiction française. On y chasse les nuages à travers le monde et l'Histoire dans une quête surréelle. Amateur de littérature

anglosaxonne et de cinéma japonais, c'est évidemment qu'on retrouve les deux pays dans son premier roman. On plonge dans l'Angleterre du XIXe siècle, et on dessine le Japon confronté à la Seconde Guerre mondiale. 
Imaginaire mondial d'après La théorie des nuages de Stéphane Audeguy

Dr. Attia Elemam Alkolaly

La théorie des nuages est un roman ambitieux car il aborde un domaine scientifique, celui de la météorologie. C'est aussi un roman original car il quitte l'autofiction et la psychologie pour raconter des histoires, mélangeant les époques (du XIXe au XXIe siècle) et les lieux (France, Angleterre, Japon, Indonésie, etc). Stéphane Audeguy y mêle des figures historiques oubliées, surtout Luke Howard, l'inventeur de la première classification des nuages, et qui inventa les noms des nuages, cumulus, stratus et cirrus; à des personnages imaginaires, tirés de l'imagination de l'écrivain, inspirés de personnages réels: le peintre Carmicheal, inspiré par Constable, les météorologues Abercrombie et Williamsson, inspirés de deux personnages bien oubliés, Abercromby et Hildebrandsson.

L'existence des passionnés de nuages, celle de Luke Howard, de Richard Abercrombie, de Virginie Latour ou d'Akira Kumo, est tout-à-fait nuageuse. Les personnages pèsent peu sur la page, ils voyagent à travers le monde qu'ils observent bien, puis vient l'heure où l'on disperse leurs cendres au vent. La veillée funèbre d'Akira Kumo aboutit à une scène de transformation du corps humain en vapeur d'eau qui rejoint le reste du monde.

Dès le Roman de la rose au Moyen Age jusqu'à Stéphane Audeguy de nos jours, le thème du ciel nuageux est complexe et énigmatique à cause de la multitude d'aspects, tirés de contextes différents, parfois contradictoires, mais souvent convergents. Ainsi, on insiste sur l'analyse logique du phénomène météorologique, sur la description des nuages comme des images de la nature qui incarnent le provisoire ainsi que le mouvement et l'instabilité. Les figures que les nuages retracent dans le ciel sont considérées comme des miroirs de l'âme, et des créations de l'imagination humaine. 
En fait, la légende d'Ixion représente, dans l'esprit occidental, la source cruciale de la pensée néphologique. Ixion s'éprend d'Héra, qu'il cherche à séduire, mais Zeus le trompe en lui envoyant un nuage, Néphélé, qui ressemble à Héra. Ixion tombe donc victime d'une illusion; il embrasse un mirage, une embrassade qui aboutit à une créature légendaire à mi-chemin entre l'homme et le cheval. Cela produit un peuple de centaures, les nubigenae "ceux qui sont nés des nuages", dont l'origine nuageuse apparaît dans leur nature hybride et leur caractère instable.

Le mythe d'Ixion, répandu dans la création artistique antique et ensuite repris par Stéphane Audeguy, suscite la confusion du phénomène des nuages. Néphélé constitue un type dans lequel se mélangent la réalité objective et la fiction subjective. Pour Stéphane Audeguy, ce ne sont pas les nuages qui l'attirent en tant que tels, mais ce que l'imagination humaine en fait. Cette image des nuages comme manifestation de la nature d'un côté, et comme résultat de la réflexion d'un autre permet aux écrivains d'exprimer l'enchaînement de la création artistique et esthétique. Ainsi, "les nuages comptent parmi les objets poétiques les plus oniriques." (Bachelard, p:212)

De son côté, Stéphane Audeguy exprime sa fascination extrême pour le thème des nuages en recourant à ce lieu commun de la littérature néphologique, et il constate que "rien au monde de plus fascinant que des nuages." Cet intérêt se manifeste clairement chez les écrivains de tous les siècles, surtout chez les poètes de l'ère baroque, du romantisme et du symbolisme. Ils désignent les nuages comme des "mystères" ou des "énigmes", comme des "merveilles" ou des "magies", comme un "secret" ou un "miracle". Citons par exemple Charles Baudelaire, qui les nomme des "magies liquides ou aériennes" (Salon de 1859 ) et de "merveilleuses constructions de l'impalpable" 
Imaginaire mondial d'après La théorie des nuages de Stéphane Audeguy

Dr. Attia Elemam Alkolaly

(Le Spleen de Paris XLIV), qui exercent un pouvoir magnétique sur l'observateur. Stéphane Audeguy remarque que les nuages représentaient, pour l'homme, un véritable "casse-tête", un défi à la logique: ils sont "de constants miracles, aériens, infatigables", "des oracles" et "des signes mystérieux". Les nuages constituent, dans le ciel, des signes énigmatiques, et des figures dessinées que l'homme essaie à déchiffrer.

De son côté, le poète romantique allemand Novalis conçoit "les nuages comme un alphabet céleste, comme une écriture dans le grand livre de la nature, que l'homme cherche à lire et à comprendre". (Guldin p:77-78)

Nous observons que la méditation des nuages dans le ciel pose l'homme dans une situation prodigieuse, une rêverie, une réflexion, un état délirant de suivre le mouvement des nuages, comme le dit Baudelaire: "Ces nuages aux formes fantastiques [...] me montèrent au cerveau comme une boisson capiteuse". (Salon de 1859) Les écrivains conçoivent les nuages comme des phénomènes énigmatiques qui ont un impact physique et psychique efficace sur l'humanité, tout en les décrivant comme le signe d'une nature floue. L'individu, en méditant les nuages, se confond avec le phénomène observé, et il devient à son tour un nuage. François-René de Chateaubriand, appelé "prince des nuées", a déclaré: "fixant mes regards au ciel, je commençais une incantation. Je montais avec ma magicienne dans les nuages [...] j'allais, au gré des tempêtes, agiter la cime des forêts, ébranler le sommet des montagnes, ou tourbillonner sur les mers [...] Je devenais le nuage" (Mémoires d'outretombe I, p:227-228) 
En fait, la phase des nuages se caractérise par trois aspects principaux, associés aux faits de l'existence humaine: le fugitif, le mouvement continuel et la transformation perpétuelle. Ceux-ci se manifestent par trois axes différents: l'axe temporel pour le fugitif, l'axe spatial pour le mouvement, et l'axe morphologique pour la transformation. De nombreux écrivains insistent sur le caractère transitionnel des nuages: "C'est un univers de formes en continuelle transformation". (Bachelard, p:222) Victor Hugo remarque que "rien ne change de forme comme les nuages" (Les rochers); et George Sand, à son tour, voit que "les nuages sont ce qu'il y a de plus changeant dans le monde" (Le nuage rose).

même, les critiques modernes qui s'occupent des nuages comme un thème littéraire et artistique observent de près les "continuelles transitions" des nuages, leur "métamorphose inlassable", et leur "transformation incessante". Ils notent également que les nuages présentent un aspect "instable", "inconsistant" et "amorphe", à cause de leurs contours "flous", "diffus", "fluides", "fluctuants", "indécis" et "ambivalents". (Damisch, 1972)

Cet intérêt résulte de l'évolution rapide de la météorologie, et des inventions scientifiques du XIXe siècle, ère à laquelle des météorologues comme Jean-Baptiste Lamarck (ou de Lamarck) en France et Luke Howard en Angleterre, par la classification des différentes formes nuageuses, présentent des méthodes fixes d'analyse de ces phénomènes fuyants. L'instabilité des figures nuageuses se manifeste à travers l'inconstance humaine, le changement du caractère et l'incertitude du destin. "L'homme peut apprendre de la nature qui il est [...] Le regard lucide saisit les mouvements des nuages. Ceux-ci tendent un miroir reflétant les comportements humains [...] Les hommes imitent les nuages versatiles." (Davy, p:177) 
Imaginaire mondial d'après La théorie des nuages de Stéphane Audeguy

Dr. Attia Elemam Alkolaly

D'après Victor Hugo, les nuages signifient la vie nomade, qui permet d'associer des idées de migration, de voyage ou d'errance, principes qui confirment les intentions philosophiques ou politiques de l'individu. De plus, les nuages sont soumis à des forces mystérieuses et au progrès scientifique. A travers les siècles, le discours religieux sur les nuages se transforme grâce à l'évolution de la météorologie. Comme le dit Stéphane Audeguy: "Les siècles passaient et la corporation des savants se confondait de moins en moins avec celle des prêtres; et plus les cieux se dépeuplaient de leurs anges, plus ils perdaient leurs prodiges et leurs dieux, plus ils s'emplissaient d'hommes embarqués dans des nacelles ou des aéroplanes." (La théorie des nuages, $p: 81$ )

En fait, avec le progrès des connaissances scientifiques météorologiques, l'image du monde a été transformée diamétralement au tournant du siècle entre les Lumières et le Romantisme naissant. Dès le début du XIXe siècle, les nuages attirent l'attention des savants: Jean-Baptiste Lamarck en France et Luke Howard en Angleterre établissent une classification méthodique de différents types nuageux. En 1802, Lamarck présente, dans son essai intitulé "Sur la forme des nuages", deux motifs essentiels de la composition des nuages: la force des vents et l'influence des rayons de soleil. De plus, il suppose, en 1805, une région des vapeurs remplie par de petites particules, qui peuvent se condenser pour former des nuages. Il présente aussi, en 1802, deux types de nuages: les" nuages groupés", qui sont des nuages de convection, et les "nuages en voile", qui incarnent des couches de nuages couvrant le ciel. En 1805, il a donné de nombreux types de nuages: "nuages brumeux, nuages terminés, nuages en voile, nuages en lambeaux, nuages moutonnés, nuages groupés ...etc..." 
Cette taxinomie implique de nombreuses lacunes car Lamarck s'occupe surtout de la forme visible des nuages (densité, contours, éclat), au lieu de s'occuper à la structure physique "des vapeurs, aux principes cachés de leur composition." (Guldin, p:152-159) Mais son génie consiste dans la volonté de "nommer les nuages, d'inventer une terminologie, qui manque malheureusement de précision scientifique, car elle imite trop le langage fleuri de l'inspiration pastorale et du calendrier révolutionnaire." (Hamblyn, p:130)

Quoi qu'il en soit, ses théories restent inaccessibles au niveau international à cause de la rareté des termes scientifiques et à cause de l'usage du français, qui empêche une diffusion mondiale de ses idées. Pourtant, la classification de Lamarck a ses acquisitions en établissant les diverses altitudes des nuages (haute, moyenne et basse).

Concernant la classification établie par Luke Howard, celui-ci s'occupe essentiellement de la forme extérieure des nuages, utilisant la notion de "modification", basée sur l'unité des divers types de nuages. Luke Howard expose trois "modifications" centrales, le cirrus, le cumulus et le stratus, et quatre "modifications" de leur fusion: deux formes intermédiaires, le "cirro-cumulus et le cirro-stratus", et deux formes combinées, le "cumulo-stratus et le cumulo-cirro-stratus". Il a donc réduit les formes nuageuses à trois types de base, qui peuvent être combinés. Ainsi, "Howard avait prêté un langage aux formes les plus ineffables et les plus riches de la nature [...] Il avait donné des noms aux nuages". (Hamblyn, p: 12)

En réalité, les noms inventés par Luke Howard sont descriptifs, simples, et inscrits en latin afin d'être à la portée de tous. C'est l'un des motifs essentiels de la diffusion internationale de ses théories. Il a choisi des termes scientifiques connus pour toute l'Europe comme genres de base cirrus "fibre" ou "cheveu", cumulus "tas" et stratus "couche". Son talent consiste dans le fait qu' "en reconnaissant la nature changeante et vagabonde des 
Imaginaire mondial d'après La théorie des nuages de Stéphane Audeguy

Dr. Attia Elemam Alkolaly

nuages, il conservait aux choses aériennes toute leur antique et sensuelle séduction, même dans le cadre d'une taxinomie empirique". (Ibid, p:166)

Par conséquent, l'étude des nuages devient, grâce à Luke Howard, une véritable discipline scientifique au cours du XIXe et XXe siècles. Actuellement, la météorologie connaît presque dix genres de nuages, dont cinq correspondent aux modifications de Luke Howard (cirrus, cumulus, stratus, cirro-cumulus, cirrostratus). De plus, ses idées exercent une influence cruciale sur les lettres et les arts romantiques. Les écrivains français réagissent-ils à cette révolution scientifique? En fait, le nombre d'écrivains français qui suivent la technique de Luke Howard est très peu. Parmi les auteurs français, "rares sont ceux qui ont fait allusion à la classification [...] Les écrivains utilisent rarement les termes techniques". (Dufour, p: 9-10)

Il s'agit soit d'un roman scientifique, soit d'un roman d'aventures exotiques, soit d'un journal de voyage. Citons Jules Verne (Le Rayon vert), Edouard Peisson (Mer baltique) ou Saint-Exupéry (Courrier Sud). Evidemment, La théorie des nuages de Stéphane Audeguy représente un cas exceptionnel. Il retrace, au début du XXIe siècle, l'histoire de la météorologie et s'occupe beaucoup à la classification scientifique des nuages. Dans la plupart des oeuvres modernes, les écrivains traitent les formes nuageuses d'une autre façon, négligeant la méthode des météorologistes. Au lieu de suivre la discipline de Lamarck ou de Luke Howard, ils adoptent un système rhétorique: la métaphore, qui vise à la méditation. Les écrivains parlent du ciel nuageux comme d'un "réseau", d'un "filet" ou d'un "appareil" de nuages. De nombreux textes abordent l'aspect maritime du ciel nuageux, une image tirée de la notion antique d'une "mer supérieure", d'une "vaste mer de l'air". 
La notion de la "mer aérienne" est attirante dans le cas des nuages observés. Stendhal, monté sur la montagne entre Cergue et Genève, admire une "immense plaine de nuages blancs [...] cette mer cotonneuse [...] d'une blancheur si éclatante vue d'en haut". (Journal, 21 octobre 1823) Stéphane Audeguy imagine Luke Howard, qui "s'est hissé au-dessus des nuages qu'il a tant scrutés, et c'est à peine d'abord s'il les reconnaît, car le ciel des nuages est devenu mer." (La théorie des nuages, p:109) Et Guy de Maupassant, dans un vol en ballon, découvre "la mer de nuages", "l'océan des nuées blanches", "des océans de vagues roulées, soulevées, immobiles et furieuses, dont l'écume luisant aveugle les yeux" (Sur les nuages).

La métaphore maritime est aussi liée à l'image des nuagesvaisseaux, des nuages-voiles et des nuages-flottilles. L'identification des nuages à des paysages ne se contente pas seulement à évoquer des lieux divers: elle comprend aussi les édifices, et toutes les activités humaines. La présentation littéraire du ciel peuplé de nuages apparaît dès le début du XIXe siècle; Victor Hugo considère les nuages comme des "peuples du ciel" (Océan) ou des "habitants du ciel" (Bernardin de SaintPierre: Harmonies de la Nature IV), comparables aux hommes sur terre. Parmi les figures animales les plus souvent utilisées, est celle du mouton. Lamarck parle de "nuages moutonnés ou attroupés". Il s'agit d'une tradition biblique, car l'espèce de nuage "floccus" désigne jusqu'au XIXe siècle "les troupeaux divins, séjournant dans les jardins célestes". (Guldin, p:83)

Les textes scientifiques et littéraires récupèrent cette image des nuages moutonneux, comme le constate Stéphane Audeguy plaisamment dans le roman: "dans les nuages de type divisé on a vu ainsi apparaître des moutons (cirrocumulus) et mêmes de gros moutons (altocumulus)"; (La théorie des nuages, $\mathrm{p}: 172$ )

Pour les écrivains, les différents animaux, les personnages, et les paysages qu'ils aperçoivent dans les nuages sont April 2016 
Imaginaire mondial d'après La théorie des nuages de Stéphane Audeguy

Dr. Attia Elemam Alkolaly

effectivement le produit de leur imagination. Le cerveau humain et les nuages s'assemblent et se ressemblent, ou comme le dit Stéphane Audeguy:

"le cerveau des hommes a la forme des nuages, et qu'ainsi les nuages sont comme le siège de la pensée du ciel; ou alors, que le cerveau est ce nuage dans l'homme qui le rattache au ciel." (p:27)

En fait, l'observateur des nuages se laisse plonger dans une rêverie profonde oubliant les objets qui l'entourent. C'est là que réside le danger si le contemplateur des nuages croit trop à ses imaginations, par lesquelles il peut toucher la folie, comme le constate Stéphane Audeguy: "Rien au monde de plus fascinant que les nuages; mais là est le danger. Car rien aussi n'est plus vain, plus trompeur, plus stupéfiant que cette matière toujours changeante, toujours renouvelée; et que l'on peut si aisément s'épuiser à vouloir décrire, comprendre, dominer [...] Le long et doux cortège des amoureux de nuages comporte [...] un peu trop de suicidés, de désespérés, d'amoureux éconduits et de solitaires tristes." (La théorie des nuages, p:309)

En fait, la fascination des personnages du roman, scientifiques ou amateurs, des nuages est dû essentiellement au hasard et à certaines situations qu'ils ne maîtrisent pas. Et on ne sait pas pourquoi Akira Kumo a choisi Virginie Latour pour classer sa bibliothèque mais, dès le début de son travail, elle s'est passionnée des nuages. Luke Howard découvre aussi les nuages par hasard, allongé sur un banc dans un jardin pendant qu'une voisine lui fait la lecture de la Bible:

"Luke Howard écoute Mariabella; la parole divine lui semble toujours aussi belle; les nuages qui passent, toujours changeants et pourtant immuables, lui paraissent chanter des cantiques lumineux et muets. Et parfois il lui semble que ce sont les nuages qui regardent passer les hommes. Il remercie la Providence 
de lui avoir fourni, en le blessant, l'occasion d'entendre ainsi le livre des livres." (p:56-57)

Pour Akira Kumo, son intérêt passionné pour les nuages est beaucoup plus énigmatique. Il est toujours obsédé par une question dérisoire: "Pourquoi s'est-il un jour pris de passion pour les nuages? Il ne sait pas [...] Mais parfois il sent que la réponse à cette question-là l'attend, tapie comme une bête inconnue dans la jungle opaque de sa mémoire; alors Akira Kumo tremble qu'elle ne bondisse et ne vienne l'anéantir, d'un seul coup." (p:58) Pour lui, sa préoccupation croissante pour les nuages ne sera levée que plus tard dans le texte, et il comprendra qu'il doit échapper au nuage atomique à Hiroshima.

Au-delà d'histoires qui relatent les nuages, La théorie des nuages met en relief un rapprochement entre le temps climatique et le temps chronologique. Il montre aussi comment un nuage atomique peut apparaître à la fois comme la délivrance et l'opposition des idéaux scientifiques espérant maîtriser les nuages. Notons quelques repères temporels dans le roman. D'abord, les fautes nombreuses dans le texte lors de sa première édition. Certaines sont des signes de temps. La plus remarquable concerne les dates de largage des deux bombes atomiques sur Hiroshima et Nagasaki, les 6 et 9 août 1945: "Pourquoi Hiroshima, et puis Nagasaki? Pourquoi une bombe le 5 août et une seconde le 6?" (La théorie des nuages, p:155) D'autres concernent l'âge de certains personnages: Richard Abercrombie, "âgé de trente-neuf ans en 1891", se trouve avoir, quelques pages plus loin et "en 1892, quarante-cinq ans". (p:254) De plus, un horaire précis est souvent cité, et il inaugure le roman: "Vers les cinq heures du soir, tous les enfants sont tristes: ils commencent à comprendre ce qu'est le temps. Le jour décline un peu. Il va falloir rentrer pourtant, être sage, et mentir. Un dimanche de juin 2005 , vers les cinq heures du soir, un couturier japonais, nommé Akira Kumo, parle à la bibliothécaire qu'il vient d'engager." (p:13) 
Imaginaire mondial d'après La théorie des nuages de Stéphane Audeguy

Dr. Attia Elemam Alkolaly

Cette phrase magistrale annonce l'importance du temps aux sens chronologique et climatique, de la même façon que le mot "théorie" du titre est à prendre dans ses deux sens. Et ce même horaire clôt la scène finale du roman quand Virginie Latour a dispersé les cendres d'Akira Kumo lors d'une tempête: "Vers les cinq heures du soir, Virginie Latour est à l'abri". (p:319) Dans le roman, cet horaire revient: "la journée passe ainsi, jusque vers les cinq heures du soir" (p:66), "vers les cinq heures du soir, ils vont se promener". (p:146)

Nous pouvons y entendre un souvenir de la fameuse phrase qui résumait le genre romanesque pour Paul Valéry: "la marquise sortit à cinq heures". Claude Mauriac, le fils de François Mauriac, en avait même fait le titre d'un de ses romans, publié en 1984. De même, deux ans après celui de Stéphane Audeguy, un jeune écrivain, Gilles Heuré, intitule encore son premier roman: L'Homme de cinq heures (2009). Les nuages ne représentent pas seulement un thème central pour les personnages, scientifiques ou amateurs, du roman. Ils sont utilisés aussi pour montrer le lien entre le temps qui passe et le temps qu'Il fait. Pour cette idée, Stéphane Audeguy déclare dans un entretien:

"Pour l'anecdote, l'idée de l'intempérie comme mode de figuration de l'histoire me vient de Queneau, même si elle ne lui est pas propre: il utilise massivement l'équivalence entre le temps climatique et le temps historique." (La Femelle du requin, p:59)

En effet, le roman de Raymond Queneau, romancier, poète et dramaturge français [1903-1976], intitulé Saint Glinglin, et paru en 1948, présente un monde sans nuages et donc sans pluie, dans lequel le temps et donc l'Histoire se sont stoppés.

Stéphane Audeguy utilise des expressions allusives pour faire un rapprochement entre temps et Temps: "les semaines filent, 
comme au ciel de Paris font les nuages d'été, précédant les orages" (La théorie des nuages, p:79), "le temps file comme le vent." (p:90) L'idée de maîtriser le Temps en contrôlant le temps se trouve chez certains personnages du roman. Si Stéphane Audeguy évoque le mépris de Napoléon pour les météorologues lors de la campagne en Russie, il montre ailleurs l'importance des études sur la direction des vents pour l'utilisation des gaz pendant la Première Guerre mondiale, et l'importance des conditions météo pour préciser la date exacte du largage de la bombe atomique à Hiroshima. L'auteur présente d'autres personnages qui s'occupent du progrès de la science météorologique. C'est le cas de Williamsson, météorologue concurrent d'Abercrombie qui lors du congrès de 1889 se projette en l'an 2000:

"Nous saurons déplacer les nuages et maîtriser les pluies [...] qui dit contrôle des nuages dit aussi bien et surtout contrôle de l'agriculture. Nous en aurons fini avec les famines chroniques des sauvages de toutes races; des champs de tomates couvriront le Sahel, des océans de blé se balanceront dans les longues plaines du Kalahari [...] Il n'y aura plus de distinction entre les régions fertiles et les régions stériles; la terre sera ce jardin où nous vivrons l'âge d'or dont nous entretiennent les mythologies et les religions, et l'homme sera bon; nos petits-enfants reverront verdir et fleurir les plaines désolées de Canaan, et ce que la foi de nos ancêtres chantait, la science de nos petits-enfants l'accomplira sur terre." (p:181-183)

Le lien entre temps et Temps se manifeste au plus haut degré à Hiroshima. Avant de larguer la bombe sur cette ville on a envoyé un "appareil de reconnaissance météorologique d'un beau grisbleu cherchait une ville sans nuages [...] Juste après que le bombardier de type B52 a largué sa charge, il y a sûrement un instant étrange pour ceux qui ont repéré ce petit point brillant qui descend lentement vers la ville, il y a un moment unique". Vraiment, C'est un instant unique dans un siècle de fer et de feu, 
Imaginaire mondial d'après La théorie des nuages de Stéphane Audeguy

Dr. Attia Elemam Alkolaly

dans le silence d'un ciel sans nuage, qui semble avoir absorbé le temps et l'espace, à l'exception de ce point brillant dans le ciel, qui descend. Ensuite cet instant disparaît dans l'oubli absolu, parce que la "bombe atomique explose, exactement à la hauteur prévue par l'état-major." (La théorie des nuages, p:157-158)

A ce moment exceptionnel, le temps s'arrête au sens propre du mot pour Akira Kumo avec l'arrêt de la montre de sa soeur Kinoko. Si Akira Kumo a été sauvé en se baignant dans un fleuve, sa soeur, sur la rive, était malchanceuse. Au moment de l'explosion, "il trouve un objet à demi fondu, qu'il connaît bien, qui est la montre de Kinoko, une petite montre allemande de gousset, qu'il se brûle à vouloir saisir". On se penche sur la rivière, et on a refroidi le métal fumant, qui brille au soleil. Le verre a disparu et "les aiguilles se sont incrustées dans le cadran; elles marquent huit heures quinze." (p:269)

Tiphaine Samoyault observe que la montre arrêtée est l'une des caractéristiques essentielles d'Hiroshima, "à jamais immobilisée sur la catastrophe, sur cet instant fatal, dont la fatalité est indiquée a posteriori par ce temps devenu un non-temps. Certaines montres s'arrêtent ainsi, non sous l'effet d'un grippement interne mais sous celui d'une déflagration soudaine qui détruit tout un temps. C'est le monde lui-même qui s'arrête." (Samoyault, p:157) Pour Akira Kumo, le temps précédent de la bombe atomique s'est aboli et devenu un passé enraciné dans sa mémoire. La date de 1933, sa date de naissance officielle, est devenue 1946. Tout était amalgamé. Ce nuage d'Hiroshima est indépendant des nuages météorologiques répandus tout au long du roman: il est unique et diamétralement contrôlé par l'homme. D'un côté, il s'inscrit dans un temps circulaire, distingué par le retour des 
nuages, car la date d'Hiroshima est presque la plus connue du XXe siècle. D'autre côté, quand l'explosion du Krakatoa est évoquée et suivie d'un nuage dévastateur, on parle alors de "ce nuage qui fut un volcan". II est finalement le symbole d'une fin du monde:

"Le livre sacré des Américains conte la fin du monde, c'est un récit de sang et de feu où l'on punit les méchants, et maintenant au-dessus de la ville se tient un nuage unique et lourd sorti tout droit de ce livre sacré, advenu sur la terre par la grâce des Américains, comme si la ville rasée flottait au-dessus d'elle-même, réduite en poussières toxiques, comme si le nuage de l'apocalypse avait absorbé toute la poussière qu'exhalait la ville." (La théorie des nuages, $\mathrm{p}: 270$ )

Cet extrait implique la religion, la foi des ancêtres et le rôle de la science dont parlait Williamsson en 1889.

Pour reprendre l'énumération de Stéphane Audeguy, on définit ce nuage artificiel comme une espèce de "nuage monstre".

C'est non seulement sa phase qui le rend exceptionnel mais aussi sa création, son itinéraire et ses répercussions: "Le nuage d'Hiroshima est un nuage très particulier, un nuage que peu d'hommes ont vu auparavant, là-bas, sur une base militaire du NouveauMexique, un nuage prolongé, jusqu'au sol, d'un pédoncule effilé, un nuage posé sur un pied comme un champignon grotesque." (Idem)

Cependant, le nuage d'Hiroshima n'est pas séparé des nuages météorologiques. C'est à cause de ce nuage qu' Akira Kumo va s'occuper des nuages pour comprendre: "Hiroshima s'est levé dans sa mémoire, comme dans un brouillard d'abord; puis le brouillard s'est évanoui." (p:258-259) Cette phrase est prononcée quand il a été sauvé du nuage d'Hiroshima, et il s'est suicidé. Ce nuage, comme celui du Krakatoa qui a évoqué un volcan, a laissé des répercussions périlleuses sur la météorologie, créant 
Imaginaire mondial d'après La théorie des nuages de Stéphane Audeguy

Dr. Attia Elemam Alkolaly

un temps particulier et ici c'est l'Histoire qui a maîtrisé le temps: "Le nuage s'est encore étendu jusqu'au point où il a semblé n'y plus tenir, et il s'est mis à pleuvoir; simplement, la pluie était noire, entièrement noire, elle rend la poussière des corps à la terre. Il pleuvra ainsi tous les soirs, pendant des semaines." (La théorie des nuages, $\mathrm{p}: 271$ )

C'est ici, dans le drame d'Hiroshima comme dans le congrès de 1889 , que la vision totalitaire du progrès scientifique qui compte. Ce nuage n'appartient plus à un temps cyclique; ce temps que les savants auraient voulu contrôler pour le rendre réglé. C'est un nuage qui ne revient pas et qui se passe dans un temps déterminé: il y a un avant et un après.

Enfin, le roman présente temps et Temps en lutte permanente, et c'est finalement le temps climatique qui semble l'emporter, comme l'indique la scène orageuse à la fin du roman, face à laquelle les hommes restent handicapés. Les histoires racontées n'empêchent pas le retour immuable des nuages qui "passent, comme à l'accoutumée, indifférents au commerce des hommes." (p: 165) Ils ne peuvent que les observer, les classer et contempler en les regardant. D'après Stéphane Audeguy, ce roman est "un roman écologique au sens très large"; il présente un panorama de l'existence des êtres vivants, dont l'être humain n'est qu'un spécimen.

Le Temps historique, avec son mouvement cyclique, et le temps climatique, avec le retour perpétuel des saisons, se combinent et se complètent pour tracer l'Histoire de l'humanité. Le début et la fin du roman se ressemblent temporellement et géographiquement: la scène finale sur la lande de Hampstead est un retour en boucle sur le lieu initial d'où Luke Howard aimait à regarder les nuages. C'est aussi un retour à l'heure à laquelle le roman avait commencé: il s'achève à "cinq heures du soir". 


\section{Bibliographie}

\section{I- $\underline{\text { Corpus: }}$}

- Audeguy, Stéphane. La théorie des nuages. Paris:

Editions Gallimard. 2005.

II- Ouvrages de critique:

- Becker, Karin. La pluie et le beau temps dans la littérature française. Hermann. 2012.

- Bachelard, Gaston. L'air et les songes. Essai sur l'imagination du mouvement. Paris: Librairie José Corti, 1948.

- Danisch, Hubert. Théorie du nuage. Pour une histoire de la peinture. Paris, Seuil. 1972.

- De Bary, Cécile. Itinéraires. Littérature, textes, cultures. "La fiction aujourd'hui". 2013.

- Dufour, Louis. Poupart, R. Les nuages dans la littérature française. Bruxelles: Institut Royal Météorologique de Belgique, 1973.

- Gottingen, Udo Schoning. "Sur la notion d'interculturalité et ses rapports avec l'histoire littéraire" in Cahiers d'Histoire des littératures. Romanes Romanististische Zeitschrift für Literaturgeschichte. 1995.

- Guldin, Rainer. Die Sprache des Himmels. Eine Geschichte des wolken. Berlin: Kadmos, 2006.

- Hamblyn, Richard. L'invention des nuages. Paris: Editions J C Lattès, 2003.

- Samoyault, Tiphaine. La montre cassée. Lagrasse: Verdier, 2004.

III- Numéros de revues:

- Le Magazine littéraire. N0. 483. Février 2009.

- Le Magazine littéraire. N0. 482. Janvier 2009.

- La Nouvelle Revue française. No. 404. Juin 2013.

- La Nouvelle Revue française. No. 585. Avril 2008.

- La Nouvelle Revue française. N0. 581. Avril 2007. 
Imaginaire mondial d'après La théorie des nuages de Stéphane Audeguy

Dr. Attia Elemam Alkolaly

- La Nouvelle Revue française. N0. 582. Juin 2007.

- Le Matricule des anges. No. 61. Mars 2005.

- Europe. N0. 916-917. Août- Septembre 2005.

IV- Entretiens de Stéphane Audeguy:

- Entretien avec Thierry Guichard dans Le

Matricule des anges. N0. 101. Mars 2009.

- Entretien in La Femelle du requin. N0. 29. Eté 2007.

V- Site internet:

- @ la lettre. Com, http://www. a la lettre. Com/ interview-stéphane- audeguy. php- 